\title{
HOMOGENEOUS WEYL CONNECTIONS OF NON-POSITIVE CURVATURE
}

\author{
GABRIELA TERESZKIEWICZ AND MACIEJ P. WOJTKOWSKI
}

\begin{abstract}
We study homogenous Weyl connections with nonpositive sectional curvatures. The Cartesian product $\mathbb{S}^{1} \times M$ carries canonical families of Weyl connections with such a property, for any Riemmanian manifold $M$. We prove that if a homogenous Weyl connection on a manifold, modeled on a unimodular Lie group, is non-positive in a stronger sense (streched non-positive), then it must be locally of the product type.
\end{abstract}

\section{Introduction}

Let $M$ be a Riemannian manifold with the metric $g$. A Weyl connection on $M$ is a linear symmetric connection with conformal parallel transport. A modern account of the theory of such connections was given by Folland, $\mathrm{F}$.

Let us recall that a Weyl connection $\widehat{\nabla}$ is determined by the 1-form $\varphi$ satisfying $\hat{\nabla}_{X} g=-2 \varphi(X) g$. The difference of two linear connections is a tensor. Denoting by $\nabla$ the Levi-Civita connection, we get

$$
\widehat{\nabla}_{X} Y=\nabla_{X} Y+\varphi(Y) X+\varphi(X) Y-\langle X, Y\rangle E,
$$

for any tangent vector fields $X, Y$, where $E$ is the vector field dual to $\varphi$, and $\langle\cdot, \cdot\rangle$ denotes the metric $g$.

For any smooth function $U$ on $M$ the pair $(g, \varphi)$ defines the same Weyl connection as the pair $\left(e^{-2 U} g, \varphi+d U\right)$. In particular for a closed 1 -form $\varphi$ the Weyl connection is locally a Levi-Civita connection of some metric in the conformal class.

The curvature operator of a Weyl connection

$$
\widehat{R}(X, Y)=\widehat{\nabla}_{X} \widehat{\nabla}_{Y}-\widehat{\nabla}_{Y} \widehat{\nabla}_{X}-\widehat{\nabla}_{[X, Y]}
$$

has the symmetric part, called the distance curvature, $\widehat{R}_{s}(X, Y)=$ $d \varphi(X, Y) I$, where $I$ is the identity operator, $[\mathbf{F}$. The anti-symmetric part of the curvature operator $\widehat{R}_{a}=\widehat{R}-\widehat{R}_{s}$, called the direction curvature, can be used to define the sectional curvature $\widehat{K}(\Pi)$ of the Weyl connection in the direction of a plane $\Pi$

$$
\widehat{K}(\Pi)=\left\langle\widehat{R}_{a}(X, Y) Y, X\right\rangle,
$$

Date: April 9, 2018.

1991 Mathematics Subject Classification. 53C24 (53C21).

The authors were partially supported by the NCN grant 2011/03/B/ST1/04427. 
for any orthonormal basis $\{X, Y\}$ of $\Pi$. These sectional curvatures depend on the choice of the Riemannian metric $g$ in the conformal class, and not on the Weyl connection alone. Indeed, although the anti-symmetric part of the curvature operator $\widehat{R}_{a}$ is independent of the choice of the metric, with the passage to the metric $e^{-2 U} g$ the sectional curvatures acquire the universal factor $e^{2 U}$. Hence the sign of sectional curvatures is well defined.

For a Weyl connection on a compact manifold Gauduchon, Gau, established the existence and uniqueness of a metric in the conformal class such that the respective vector field $E$ is divergence free, divE $=$ 0 . We will say in such a case that the pair $(g, E)$ is the Gauduchon representation of the Weyl connection, and $g$ is the Gauduchon metric, irrespective of the manifold $M$ being compact or not.

It was observed in [W1, W2] that Weyl connections are related to a special class of dynamical systems called Gaussian thermostats. The paper of Gallavotti and Ruelle, G-R, gives an account of the physical significance of these systems. A Gaussian thermostat is defined by a Riemannian metric on a manifold $M$ and a vector field $E$. The trajectories of the Gaussian thermostat satisfy the following ordinary differential equations in the tangent bundle $T M$, W1].

$$
\frac{d}{d t} x=v, \nabla_{v} v=E-\frac{\langle E, v\rangle}{\langle v, v\rangle} v
$$

where $x=x(t) \in M$ is a parametrized curve in $M$. By the force of these equations the "kinetic energy" $v^{2}$ is constant. Fixing the value of this constant $k=v^{2}$ we obtain that the trajectories of (1) are geodesics of the Weyl connection defined by the vector field $k^{-1} E$. (Note that this coincidence does not extend to the parametrizations: in general the natural parameter of the Weyl geodesic is not proportional to the arc length.)

We can see that the behavior of the trajectories of a Gaussian thermostat for different values of the kinetic energy is governed by the family of Weyl connections defined by the family of vector fields $\gamma E, \gamma>0$, where large values of $\gamma$ correspond to small values of the kinetic energy.

The goemetry and dynamics of multidiemsional Gaussian thermostats were studied recently in the paper of Daraibekov and Paternain, [D-P].

In the present paper we study Weyl connections with non-positive sectional curvatures, the non-positive Weyl connections. The motivation comes from the results of [W1, W2, P-W]. In the Riemannian case if all the sectional curvatures are negative then the geodesic flow is an Anosov system. It was proven in [W1 that negative sectional curvatures of the Weyl connection lead to some hyperbolic properties, namely the dominated splitting with exponential growth and decay of volumes. A simplified account of this result can be found in [P-W]. 
In particular in dimension 2 we get the Anosov property. In this case there is only one sectional curvature and it is equal to the Gaussian curvature of the Gauduchon metric. Surfaces with negative Gaussian curvature are well understood. For any divergence free vector field $E$ on such a surface we get the Gaussian thermostat with the Anosov property.

In dimension 3 and higher we do not know of examples of Weyl connections with negative sectional curvatures, beyond the small perturbations of Levi-Civita connections with negative sectional curvatures.

In the search of such examples one encounters rigidity phenomena similar to the Riemannian case.

In the work of Alekseevski[Al], Heintze [He], and Azencott and Wilson [Az-W], the homogeneous Riemannian metrics with non-positive sectional curvatures were classified. In particular it was found that left invariant metrics on unimodular Lie groups are by necessity flat.

Constant vector fields on flat tori give examples of non-positive Weyl connections. It follows from the results in W3 that there are no other non-positive Weyl connections on tori with the flat Gauduchon metric. Moreover there are partial results in [W3] towards the conjecture that also for arbitrary Gauduchon metrics there are no other non-positive Weyl connections on tori. For example it was proven in [W2] that there are no locally Riemannian non-positive Weyl connections on tori except for the ones defined by constant vector fields. Weyl connections on $\mathbb{S}^{1} \times \mathbb{S}^{n-1}$ were also studied in [W3. In the present paper we generalize these results.

The examples in W3 have the additional properties that the vector field $E$ is parallel, i.e., $\nabla E=0$, and it is stretched non-positive

$(S N P)$ there is $\gamma_{0} \geq 0$ such that for every $\gamma \geq \gamma_{0}$ the Weyl connection defined by $\gamma E$ is non-positive.

It is straightforward that any parallel vector field $E$ is stretched nonpositive (cf. Proposition 2.3).

Note that the stretched non-positivity of a vector field $E$ is a property of the Gaussian thermostat rather than the respective Weyl connection. Indeed for two different representations $\left(g_{1}, E_{1}\right),\left(g_{2}, E_{2}\right)$ of the same Weyl connection the families $\gamma E_{1}$ and $\gamma E_{2}$ define in general different families of Weyl connections. We do not know under what conditions the two families share non-positivity for large values of $\gamma$. We will not address such issues in this paper.

Nevertheless, in the case of a compact manifold we define a stretched non-positive Weyl connection by the requirement that in the Gauduchon representation $(g, E)$ (div $E=0)$, the field $E$ is SNP.

Let us note here the paper of Narita, [N], where the Gauduchon representation is used to define Weyl connections with constant sectional curvatures. 
In Section 2 we consider Weyl connections defined by Killing vector fields, especially the non-positive connections. Parallel vector fields play an important role in this section. As a bi-product of this discussion we obtain a notable restriction on the Clifford isometries on manifolds with some non-positive sectional curvature (Proposition 2.2).

In Section 3 we study the notion of stretched non-positivity (SNP) for vector fields, and for Weyl connections, on general Riemannian manifolds.

In Section 4 homogeneous Weyl connections on Lie groups are introduced, with low dimensional examples. We exhibit an isolated nonpositive Weyl connection on SOL, [T], and SNP Weyl connections on groups with the euclidean metric (unimodular), and with the hyperbolic metric (nonunimodular).

Section 5 contains results about multidimensional examples of solvable extensions of abelian groups. We obtain there families of examples of isolated non-positive Weyl connections. It would be interesting to classify this type of geometric objects.

In Section 7 we present our main result which says that the only homogeneous SNP vector fields (or Weyl connections) modeled on unimodular Lie groups are parallel.

Our result should be compared with the Riemannian case where, on one hand, there are no non-positive, non-flat, Levi-Civita connections on unimodular Lie groups. On the other hand there are many homogeneous metrics with negative sectional curvature on manifolds modeled on unimodular Lie groups.

In Section 6 we introduce the homogeneous Weyl connections of a fairly general type. They were considered previously in the paper by Kerr, $[\mathrm{Ke}$. We will follow here the notations of the book by Besse, $\mathrm{Be}]$. Let $G$ be a Lie group and $H$ its compact subgroup. Let $\mathfrak{g}=\mathfrak{h} \oplus \mathfrak{p}$ be an $A d(H)$ invariant splitting of the Lie algebra $\mathfrak{g}$ of $G$, into the Lie algebra $\mathfrak{h}$ of $H$ and a complementary linear subspace $\mathfrak{p}$. Note that $\mathfrak{p}$ is not a Lie subalgebra in general. Any choice of an $A d(H)$ invariant scalar product in $\mathfrak{p}$ gives rise to a $G$ invariant metric on the homogeneous space $M=G / H$. We consider the subspace

$$
\mathfrak{p}_{0}=\{X \in \mathfrak{p} \mid[Y, X]=0 \quad \forall Y \in \mathfrak{h}\},
$$

on which the action of $A d(H)$ is trivial. We choose a left invariant vector field $E$ defined by an element from $\mathfrak{p}_{0}$. It projects onto $M=$ $G / H$ as a $G$-invariant vector field. The subspace $\mathfrak{p}_{0}$ may be equal to zero, however for $\mathfrak{h}=0$ we have that $\mathfrak{p}_{0}=\mathfrak{g}$. The last case is that of a left invariant metric on $G$, with a left invariant vector field $E$.

Our main result says

Theorem 7.1. If the Lie group $G$ is unimodular and a $G$-invariant vector field on $M=G / H$, defined by $E \in \mathfrak{p}_{0}$, is SNP then the vector field is parallel. 
We include in Section 6 a derivation of the formula for the sectional curvature of a homogeneous Riemannian metric, using the Jacobi fields. This derivation clarifies the calculations appearing in the proof of the main result in Section 7 .

Throughout the paper we will assume that the dimension of $M$ is at least 3. In dimension 2 for a Gauduchon representation the Weyl connection is non-positive if and only if the Gauss curvature of the metric is non-positive (see the formula (4) for the sectional curvature of the Weyl connection in Section 2).

\section{Killing fields AND NON-POSitive Weyl CONNECTiOnS}

Let us consider a Killing field $E$ on a Riemannian manifold $M$, i.e. for every vector fields $X$ and $Y$

$$
\left\langle\nabla_{X} E, Y\right\rangle+\left\langle\nabla_{Y} E, X\right\rangle=0 .
$$

Proposition 2.1. For a Killing vector field $E$ the following are equivalent

(i) E has constant length,

(ii) $\nabla_{E} E=0$,

(iii) integral curves of $E$ are geodesics.

Proof. If $E$ is a Killing vector field then for any vector field $X$ we have

$$
\nabla_{X} E^{2}=2\left\langle\nabla_{X} E, E\right\rangle=-2\left\langle\nabla_{E} E, X\right\rangle .
$$

It shows the equivalence of (i) and (ii).

If (iii) holds then we have for some positive smooth function $f$ $\nabla_{E}(f E)=0$. It follows that $\nabla_{E} E$ is parallel to $E$. However for a Killing vector field $\nabla_{E} E$ must be orthogonal to $E$. Hence (ii) follows.

Killing vector fields with constant length define one parameter groups of Clifford isometries, i.e. isometries which move all points by the same distance. It is well known that there are no Clifford isometries on manifolds of negative curvature (reference?). We have further

Proposition 2.2. Let $E$ be a Killing vector field of constant length on a Riemannian manifold $M$. If at every point of $M$ the sectional curvatures of all planes containing $E$ are non-positive, then all these sectional curvatures must be actually equal to zero and for every vector field $X, \nabla_{X} E=0$.

Proof. Let us assume for simplicity that $E^{2}=1$. Denote by $\psi^{t}, t \in \mathbb{R}$, the one parameter group of isometries generated by $E$. By Proposition 2.1 the integral curves of $E$ are geodesics and further $\psi^{t}$ shifts points by the distance $t$ along these geodesics. Let us consider a family of such geodesics $\gamma(t, u)$ where $t \in \mathbb{R}$ is the arc length parameter along a 
geodesic, and $u \in(-\epsilon, \epsilon)$ is the parameter of the family. We get the Jacobi field $J(t)=\frac{\partial}{\partial u} \gamma(t, u)_{\mid u=0}$. Since $\psi^{t_{1}} \gamma\left(t_{2}, u\right)=\gamma\left(t_{1}+t_{2}, u\right)$ we get the invariance of the Jacobi field under the flow $\psi^{t}$, i.e., $D \psi^{t} J(0)=J(t)$. Hence the Jacobi field has constant length.

On the other hand it follows from the Jacobi equations that

$$
\nabla_{E} J=Y=\nabla_{J} E \quad \text { and } \quad \frac{d^{2}}{d t^{2}} \frac{1}{2} J^{2}=Y^{2}-k J^{2}
$$

where $k$ denotes the sectional curvature of the plane spanned by $E$ and $J$. Since the Jacobi field has constant length the last expression must be equal to zero, i.e., $Y^{2}=k J^{2}$. Under the assumption $k \leq 0$ we conclude that $Y=0$ and $k=0$ everywhere.

We say that a vector field $E$ is parallel if for every vector field $X$, $\nabla_{X} E=0$. It is well known that the presence of a parallel field forces the local Cartesian product structure of $M$, see $[\mathrm{K}-\mathrm{N}]$, Chapter 99. Because of the crucial role it plays in our discussion we include a simple direct argument.

Proposition 2.3. If a vector field $E$ is parallel then its orthogonal distribution is integrable and the Riemannian manifold has locally the Cartesian product structure of an orthogonal section of $E$ by an integral curve of $E$. In particular the curvature operator $R(X, E)=0$ for any vector field $X$.

Proof. By the assumption the field $E$ is parallel along any curve. Hence the parallel transport along any curve preserves also the orthogonal complement of $E$. It follows that for $X$ and $Y$, two vector fields orthogonal to $E$, the derivative $\nabla_{X} Y$ is orthogonal to $E$. Finally the Lie bracket $[X, Y]=\nabla_{X} Y-\nabla_{Y} X$ is orthogonal to $E$. By the Frobenius Theorem the orthogonal distribution is integrable.

Let us consider a local orthogonal section $N$ of the flow of $E$, i.e., a local integral submanifold of the orthogonal distribution. Since the isometries in the flow translate points from $N$ along the geodesics by a constant distance, it follows that locally the Remannian metric is isometric to that of the Cartesian product $(-\epsilon, \epsilon) \times N$.

The last claim follows immediately from the product structure.

It is clear that conversely for the Cartesian product $N \times \mathbb{R}$ the "vertical" vector field of constant length is parallel.

As described in the Introduction, we will call a Weyl connection non-positive if it has only non-positive sectional curvatures.

For a Weyl connection defined by a vector field $E$ the sectional curvature $\widehat{K}=\widehat{K}(\Pi)$ in the direction of a plane $\Pi$ is equal to $([\mathrm{W} 1,[\mathrm{P}-\mathrm{W}])$

$$
\widehat{K}(\Pi)=K(\Pi)-E_{\perp}^{2}-\operatorname{div}_{\Pi} E,
$$


where $E_{\perp}$ is the component of $E$ orthogonal to the plane $\Pi$ and the partial divergence div ${ }_{\Pi} E$ is equal to

$$
\operatorname{div}_{\Pi} E=\left\langle\nabla_{X} E, X\right\rangle+\left\langle\nabla_{Y} E, Y\right\rangle,
$$

for any orthonormal frame $(X, Y)$ of the plane $\Pi$. Moreover $K(\Pi)$ denotes the Riemannian sectional curvature in the plane $\Pi$.

Let us assume that the field $E$ is a Killing vector field. In such a case the formula (4) reads $\widehat{K}(\Pi)=K(\Pi)-E_{\perp}^{2}$, and for planes $\Pi \ni E$ we have $\widehat{K}(\Pi)=K(\Pi)$. Hence to get a non-positive Weyl connection defined by a Killing vector field $E$ we have to have $K(\Pi) \leq 0$ for every plane $\Pi \ni E$. If we assume further that $E$ has constant length we conclude by Proposition 2.3 that $E$ is parallel and the Riemannian manifold has locally the Cartesian product structure. Conversely, parallel vector fields always have the SNP property. Namely we have the following

Proposition 2.4. For a unit parallel vector field $E$ the Weyl connection defined by $\gamma E$ is non-positive for

$\gamma^{2} \geq \max \{K(\Pi) \mid \Pi$ orthogonal to $E\}$.

We will be using several times the following formulas

Lemma 2.5. Let $X, Y_{1}, Y_{2}$ be mutually orthogonal (locally defined) unit vector fields on $M$. For a plane $\Pi$ spanned by $a X+b Y_{1}, a^{2}+b^{2}=1$ and $Y_{2}$, the Riemannian sectional curvature $K(\Pi)$ in the direction of $\Pi$ is equal to

$$
\begin{aligned}
& K(\Pi)=\left\langle R\left(Y_{2}, a X+b Y_{1}\right)\left(a X+b Y_{1}\right), Y_{2}\right\rangle \\
= & a^{2}\left\langle R\left(Y_{2}, X\right) X, Y_{2}\right\rangle+b^{2}\left\langle R\left(Y_{2}, Y_{1}\right) Y_{1}, Y_{2}\right\rangle+2 a b\left\langle R\left(Y_{2}, X\right) Y_{1}, Y_{2}\right\rangle .
\end{aligned}
$$

Further for a smooth function $f$ and the vector field $E=f X$ its partial divergence div $_{\Pi} E$ in the direction of $\Pi$, is equal to

$$
\begin{aligned}
& \operatorname{div}_{\Pi} E=\left\langle\nabla_{a X+b Y_{1}}(E), a X+b Y_{1}\right\rangle+\left\langle\nabla_{Y_{2}}(E), Y_{2}\right\rangle= \\
& a^{2}\left(\left\langle\nabla_{X} E, X\right\rangle+\left\langle\nabla_{Y_{2}} E, Y_{2}\right\rangle\right)+b^{2}\left(\left\langle\nabla_{Y_{1}} E, Y_{1}\right\rangle+\left\langle\nabla_{Y_{2}} E, Y_{2}\right\rangle\right) \\
& +a b\left(\left\langle\nabla_{X} E, Y_{1}\right\rangle+\left\langle\nabla_{Y_{1}} E, X\right\rangle\right)= \\
& a^{2}\left(d f(X)+f\left\langle\nabla_{Y_{2}} X, Y_{2}\right\rangle\right)+b^{2}\left(f\left\langle\nabla_{Y_{1}} X, Y_{1}\right\rangle+f\left\langle\nabla_{Y_{2}} X, Y_{2}\right\rangle\right) \\
& +a b\left(f\left\langle\nabla_{X} X, Y_{1}\right\rangle+d f\left(Y_{1}\right)\right)
\end{aligned}
$$

Proof. (Proposition 2.4) Let us consider three mutually orthogonal unit vectors $E, Y_{1}, Y_{2}$. We consider the Riemannian sectional curvature $K(\Pi)$ in the direction of the plane $\Pi$ spanned by $Y_{2}$ and $a E+b Y_{1}, a^{2}+$ $b^{2}=1$. Since for any vector field $Y$ the curvature operator $R(Y, E)=0$ we have by Lemma 2.5

$$
\begin{aligned}
K(\Pi)= & a^{2}\left\langle R\left(Y_{2}, E\right) E, Y_{2}\right\rangle+b^{2}\left\langle R\left(Y_{2}, Y_{1}\right) Y_{1}, Y_{2}\right\rangle+ \\
& a b\left(\left\langle R\left(Y_{2}, E\right) Y_{1}, Y_{2}\right\rangle+\left\langle R\left(Y_{2}, Y_{1}\right) E, Y_{2}\right\rangle\right. \\
= & b^{2}\left\langle R\left(Y_{2}, Y_{1}\right) Y_{1}, Y_{2}\right\rangle .
\end{aligned}
$$


Hence the curvature of the Weyl connection defined by $\gamma E$ is equal to

$$
\widehat{K}(\Pi)=b^{2}\left\langle R\left(Y_{2}, Y_{1}\right) Y_{1}, Y_{2}\right\rangle-b^{2} \gamma^{2}
$$

Let us now assume that a compact manifold $M$ possesses a parallel vector field $E$. We will look for non-positive Weyl connections other than the ones described in Proposition 2.4. First of all if we multiply $E$ by a function $f$ then we loose the non-positivity unless the function is constant.

Proposition 2.6. For a unit parallel vector field $E$ on a compact manifold and a non-constant smooth function $f$ the Weyl connection defined by $f E$ has some positive sectional curvatures.

Proof. As in the proof of Proposition 2.4 we calculate the sectional curvature of the plane $\Pi$ spanned by $a E+b Y_{1}$ and $Y_{2}$. We have further by Lemma $2.5 \operatorname{div}_{\Pi}(f E)=a^{2} \nabla_{E} f+a b \nabla_{Y_{1}} f$. Hence

$$
\widehat{K}(\Pi)=b^{2}\left(\left\langle R\left(Y_{2}, Y_{1}\right) Y_{1}, Y_{2}\right\rangle-f^{2}\right)-a^{2} \nabla_{E} f-a b \nabla_{Y_{1}} f .
$$

If the connection is non-positive then $\nabla_{E} f$ is non-negative everywhere. However for any vector field $E$ with zero divergence on a compact manifold and any smooth function $f$ the integral of $\nabla_{E} f$ is zero. It follows that if the connection is non-positive then $\nabla_{E} f$ is equal to zero.

Now non-positivity forces also $\nabla_{Y_{1}} f=0$ everywhere, for any vector field $Y_{1} \perp E$. Hence the function $f$ is constant.

For a Riemannian manifold with a unit parallel field $E$ the Ricci curvature $\operatorname{Ric}(a E+b Y)=b^{2} \operatorname{Ric}(Y)$, for any unit vector field $Y \perp E$, $a^{2}+b^{2}=1$. If we assume that the Ricci curvature is positive except for $E$ then the only non-positive Weyl connections are those described in Proposition 2.4.

Proposition 2.7. If for a compact manifold $M$ with a unit parallel vector field $E$ the Ricci curvature $\operatorname{Ric}(Y)>0$ for every unit vector $Y \perp E$ then the only non-positive Weyl connections on $M$ are those defined by $\gamma E$, where $\gamma^{2} \geq \max _{\Pi \perp E} K(\Pi)$.

Proof. Let $F$ be a vector field defining a non-positive Weyl connection. For a plane $\Pi$ spanned by $F$ and a unit vector $Y \perp F$

$$
\widehat{K}(\Pi)=K(\Pi)-\left\langle\nabla_{X} F, X\right\rangle-\left\langle\nabla_{Y} F, Y\right\rangle \leq 0,
$$

where $X=\frac{1}{|F|} F$. Adding the above inequalities over an orthogonal basis $\left(Y_{1}, Y_{2}, \ldots, Y_{n-1}, F\right)$ we obtain

$$
\operatorname{Ricc}(X)-\operatorname{div} F-(n-2)\left\langle\nabla_{X} F, X\right\rangle \leq 0 .
$$

This inequality is meaningful at every point where $F \neq 0$. Multiplying the inequality by $|F|^{n-2}$ we arrive at

$$
|F|^{n-2} \operatorname{Ricc}(X)-|F|^{n-2} \operatorname{div} F-(n-2)|F|^{n-2}\left\langle\nabla_{X} F, X\right\rangle \leq 0,
$$


which holds everywhere on $M$, with the first term vanishing where $F=0$.

Let us introduce the vector field $V=|F|^{n-2} F$. For $n \geq 3$ the field $V$ is at least $C^{1}$ and $\operatorname{div} V=|F|^{n-2} \operatorname{div} F+(n-2)|F|^{n-2}\left\langle\nabla_{X} F, X\right\rangle$ so that the last inequality reads

$$
|F|^{n-2} \operatorname{Ricc}(X)-\operatorname{div} V \leq 0 .
$$

Integrating the last inequality over $M$ we obtain

$$
\int|F|^{n-2} \operatorname{Ricc}(X) \leq 0 .
$$

Since $\operatorname{Ricc}(X) \geq 0$ we conclude that at points where $F \neq 0$ the Ricci curvature $\operatorname{Ricc}(X)=0$, and hence $F$ is parallel to $E$. By Proposition 2.6 the vector field $F$ must be a constant multiple of $E$.

Proposition 2.7 can be applied to the Cartesian product $\mathbb{S}^{1} \times \mathbb{S}^{n-1}$, and we recover the result from [W3].

\section{StretCheD NON-POSITIVITY}

For a given vector field $E$ we consider the family of Weyl connections defined by vector fields $\gamma E, \gamma>0$. For different choices of the background metric we get different families of Weyl connections.

Definition 3.1. A vector field $E$ is called stretched non-positive (SNP) if there is $\gamma_{0} \geq 0$ such that the Weyl connections defined by the fields $\gamma E$ are non-positive for all $\gamma \geq \gamma_{0}$.

A Weyl connection on a compact Riemannian manifold $M$ is called stretched non-positive (SNP) if its Gauduchon representation E is SNP.

We have established in Proposition 2.4 that a parallel vector field is SNP.

Our first task is to describe properties of vector fields $E$, and Weyl connections, with the SNP property.

Proposition 3.1. If a Weyl connection defined by a divergence free vector field $E$ on a compact manifold is SNP then

(W1) $K(\Pi) \leq 0$ for every plane $\Pi \ni E$, where $E \neq 0$;

(W2) $\left\langle\nabla_{Y} E, Y\right\rangle=0$ for every $Y \perp E$, where $E \neq 0$;

(W3) $E$ has constant length on its integral curves.

This Proposition gives us the motivation to restrict our attention to divergence free vector fields with constant length, for example $E^{2}=1$. For such unit vector fields we have

Proposition 3.2. If a unit divergence free vector field $E$ is SNP then the following conditions are satisfied

(W1) $K(\Pi) \leq 0$ for every plane $\Pi \ni E$,

(W2) $\left\langle\nabla_{Y} E, Y\right\rangle=0$ for every $Y \perp E$,

(W4) $\left\langle\nabla_{E} E, Y_{1}\right\rangle^{2} \leq 4 K\left(E, Y_{2}\right)$ for every orthonormal frame $\left(E, Y_{1}, Y_{2}\right)$. 
If a unit divergence free vector field $E$ on a compact manifold satisfies the conditions (W1), (W2) and

(W5) $\left\langle\nabla_{E} E, Y_{1}\right\rangle^{2}<-4 K\left(E, Y_{2}\right)$ for every orthonormal frame $\left(E, Y_{1}, Y_{2}\right)$ then the vector field is SNP.

Since the proofs of these two Propositions involve the same formulas we will give an amalgamated proof.

Proof. First we prove Proposition 3.1. Let $X=\frac{E}{|E|}$ where $E \neq 0$. For unit mutually orthogonal vectors $X, Y_{1}, Y_{2}$ we consider the plane $\Pi$ spanned by $a X+b Y_{1}, a^{2}+b^{2}=1$, and $Y_{2}$. Using Lemma 2.5 we get for the Weyl connection defined by $\gamma E$

$$
\begin{aligned}
& \widehat{K}(\Pi)=a^{2}\left(\left\langle R\left(Y_{2}, X\right) X, Y_{2}\right\rangle-\gamma\left(\left\langle\nabla_{X} E, X\right\rangle+\left\langle\nabla_{Y_{2}} E, Y_{2}\right\rangle\right)\right)+ \\
& b^{2}\left(\left\langle R\left(Y_{2}, Y_{1}\right) Y_{1}, Y_{2}\right\rangle-\gamma\left(\left\langle\nabla_{Y_{1}} E, Y_{1}\right\rangle+\left\langle\nabla_{Y_{2}} E, Y_{2}\right\rangle\right)-\gamma^{2} E^{2}\right) \\
+ & a b\left(2\left\langle R\left(Y_{2}, X\right) Y_{1}, Y_{2}\right\rangle-\gamma\left(\left\langle\nabla_{X} E, Y_{1}\right\rangle+\left\langle\nabla_{Y_{1}} E, X\right\rangle\right)\right) .
\end{aligned}
$$

If the quadratic form (6) is negative semi-definite then the coefficient with $a^{2}$ must be non-positive for all $\gamma \geq \gamma_{0}$. It follows that

$$
\left\langle\nabla_{X} E, X\right\rangle+\left\langle\nabla_{Y_{2}} E, Y_{2}\right\rangle \geq 0
$$

for every $Y_{2}$ orthogonal to $E$. Summing the last inequality over an orthonormal basis in the subspace orthogonal to $E$, we get

$$
(n-2)\left\langle\nabla_{X} E, X\right\rangle+\operatorname{div} E \geq 0 .
$$

Since $\operatorname{div} E=0$ we conclude that $\left\langle\nabla_{X} E, X\right\rangle \geq 0$. This inequality holds where $E \neq 0$, but it implies the inequality $\left\langle\nabla_{E} E, E\right\rangle \geq 0$ which holds everywhere. However the last function is the derivative of a smooth function $\nabla_{E}\left(\frac{1}{2} E^{2}\right) \geq 0$. Since $\operatorname{div} E=0$ the integral of the derivative over the whole manifold must vanish. It follows that $\left\langle\nabla_{X} E, X\right\rangle=0$ and $\left\langle\nabla_{Y} E, Y\right\rangle \geq 0$ for any $Y \perp E$. However since div $E=0$ we get further that $\left\langle\nabla_{Y} E, Y\right\rangle=0$ for any $Y \perp E$. This gives us (W3) and (W2).

Now the coefficient with $a^{2}$ is just the sectional curvature of the plane through $E$ and $Y_{2}$, and hence it must be non-positive, for every $Y_{2}$ orthogonal to $E$. This gives us (W1).

To prove Proposition 3.2 we consider an orthonormal frame $E, Y_{1}, Y_{2}$, and a plane plane $\Pi$ spanned by $a E+b Y_{1}, a^{2}+b^{2}=1$, and $Y_{2}$. Since we assume the vector field $E$ to have constant length 1 the formula 6 simplifies to

$$
\begin{aligned}
& \widehat{K}(\Pi)=a^{2}\left(\left\langle R\left(Y_{2}, X\right) X, Y_{2}\right\rangle-\gamma\left\langle\nabla_{Y_{2}} E, Y_{2}\right\rangle\right)+ \\
& b^{2}\left(\left\langle R\left(Y_{2}, Y_{1}\right) Y_{1}, Y_{2}\right\rangle-\gamma\left(\left\langle\nabla_{Y_{1}} E, Y_{1}\right\rangle+\left\langle\nabla_{Y_{2}} E, Y_{2}\right\rangle\right)-\gamma^{2}\right) \\
+ & a b\left(2\left\langle R\left(Y_{2}, X\right) Y_{1}, Y_{2}\right\rangle-\gamma\left\langle\nabla_{X} E, Y_{1}\right\rangle\right) .
\end{aligned}
$$

If the quadratic form (7) is negative semi-definite for all $\gamma \geq \gamma_{0}$, then in particular the coefficient with $a^{2}$ must be non-positive. It follows 
that

$$
\left\langle\nabla_{Y_{2}} E, Y_{2}\right\rangle \geq 0
$$

for every $Y_{2}$ orthogonal to $E$. Summing the last inequality over an orthonormal basis in the subspace orthogonal to $E$, we get div $E \geq 0$. Since $E$ is actually a divergence free vector field we conclude that the terms must be zero before the summation. The condition (W2) follows.

Taking this into account we obtain the following expression for the quadratic form (7)

$$
\begin{aligned}
& \widehat{K}(\Pi)=a^{2} K\left(E, Y_{2}\right)+b^{2}\left(K\left(Y_{1}, Y_{2}\right)-\gamma^{2}\right)+ \\
& a b\left(2\left\langle R\left(Y_{2}, E\right) Y_{1}, Y_{2}\right\rangle-\gamma\left\langle\nabla_{E} E, Y_{1}\right\rangle\right) .
\end{aligned}
$$

Now the coefficient with $a^{2}$ is just the sectional curvature of the plane through $E$ and $Y_{2}$, and hence it must be non-positive, for every $Y_{2}$ orthogonal to $E$. This gives us (W1).

Taking into account that the discriminant of the form must be nonpositive for all $\gamma \geq \gamma_{0}$ we obtain the condition (W4).

Conversely if the condition (W5) is satisfied then the discriminant is positive for all $\gamma \geq \gamma_{0}$. (Note that this calculation is done pointwise and hence the discriminant is considered on the compact grassmanian of tangent planes.) Together with conditions (W1) and (W2), this establishes that the unit vector field $E$ is SNP.

It follows from the formula (7) that

Proposition 3.3. If a unit vector field $E$ satisfies $\left\langle\nabla_{Y} E, Y\right\rangle>0$ for every $Y \perp E$ then the field $E$ is $S N P$.

\section{Homogeneous Weyl CONNECTiOns ON GROUPS}

Let $G$ be a Lie group and $\mathfrak{g}$ its Lie algebra. We endow the group with a left invariant metric. We will denote the respective scalar product by $<\cdot, \cdot>$.

We have the following characterization of left invariant vector fields which are parallel or Killing.

Proposition 4.1. A left invariant vector field $E$ is a Killing vector field if and only if $a d_{E}$ is skew symmetric.

A left invariant vector field $E$ is parallel if and only if $a d_{E}$ is skewsymmetric and $E$ is orthogonal to the commutator subalgebra $[\mathfrak{g}, \mathfrak{g}]$.

Proof. For left invariant vector fields $E, Y_{1}, Y_{2}$ we have

$$
\left\langle\nabla_{Y_{1}} E, Y_{2}\right\rangle=\frac{1}{2}\left(\left\langle\left[Y_{1}, E\right], Y_{2}\right\rangle+\left\langle\left[Y_{2}, E\right], Y_{1}\right\rangle+\left\langle\left[Y_{2}, Y_{1}\right], E\right\rangle\right) .
$$

This formula shows that $a d_{E}$ has the same symmetric part as the operator $Y \mapsto \nabla_{Y} E$. Hence $E$ is a Killing field if and only if $a d_{E}$ is skew-symmetric. 
Also the rest of the Proposition follows immediately from this formula.

Note also that a left invariant vector field $E$ is locally a gradient of a smooth function (i.e., the form $\varphi=\langle E, \cdot\rangle$ is closed) if and only if $E$ is orthogonal to the commutator subalgebra $[\mathfrak{g}, \mathfrak{g}]$.

For a left invariant vector field $E$ we consider the Weyl connection defined by the field.

We will study left invariant vector fields $E \in \mathfrak{g}$ which are SNP, without requiring that div $E=0$. Let us remind the reader that this is the property of the Gaussian thermostat, rather then the Weyl connection, as discussed in the Introduction. However if the field can be factored on a compact manifold by a discrete subgroup of isometries then by necessity div $E=0$, and the respective Weyl connection is SNP.

Let us recall that a Lie group is called unimodular if all left invariant vector fields are divergence free.

First of all let us consider the 3 dimensional case of unimodular groups. All the metrics on such groups can be parametrized by three parameters $\lambda_{1}, \lambda_{2}, \lambda_{3}$, so that for an orthonormal basis $\left(e_{1}, e_{2}, e_{3}\right)$ in $\mathfrak{g}$ we have

$$
\left[e_{2}, e_{3}\right]=\lambda_{1} e_{1},\left[e_{3}, e_{1}\right]=\lambda_{2} e_{2},\left[e_{1}, e_{2}\right]=\lambda_{3} e_{3} .
$$

The derivation of this can be found in [Mi].

We searched this class of examples for non-positive and SNP homogeneous Weyl connections. Assuming that not all $\lambda^{\prime} s$ are zero we found one SNP example and one isolated non-positive example. Both occur in the special case of solvable groups $\lambda_{3}=0$.

The SNP example occurs when $\lambda_{1}=\lambda_{2}$ and $E=e_{3}$. In this case the metric is flat and the vector field is parallel, see Theorem 4.1 [Mi]. Up to a permutation of indices this is the only case of an SNP vector field on a 3 dimensional unimodular group. It can be verified easily using our main result (see Theorem 4.3 below), which implies that on a unimodular group the only SNP left invariant vector fields are parallel. On the other hand we found direct calculations prohibitively cumbersome.

The isolated non-positive example occurs for the solvable group (SOL) when $\lambda_{1}=-\lambda_{2}=\lambda$ and $E= \pm \lambda e_{3}$. The vector field $E$ cannot be perturbed to any other left invariant field on SOL defining a non-positive Weyl connection. We will establish this fact in Section 5 in a more general multidimensional setting.

Due to cumbersome formulas we were unable to establish that, apart from the flat case, SOL is indeed the only 3 dimensional unimodular Lie group with a non-positive homogeneous Weyl connection. We conjecture that this is so. 
Let us further consider all 4 dimensional extensions $\mathfrak{h}$ of the above 3 dimensional unimodular Lie algebra $\mathfrak{g} \subset \mathfrak{h}$. We want to check the nonpositivity properties of the field $E=b \in \mathfrak{h}$, where $b$ is the unit vector orthogonal to $\mathfrak{g}$. The extension is completely described by the operator $a d_{b}=L$. However the operator is not arbitrary. The Lie algebra $\mathfrak{h}$ is unimodular if and only if $L \mathfrak{g} \subset \mathfrak{g}$ (or equivalently $[\mathfrak{h}, \mathfrak{h}] \subset \mathfrak{g}$ ).

By direct calculation we obtain that the Jacobi identity implies the following

Proposition 4.2. For the diagonal operator $\Lambda$ with eigenvalues

$1, \lambda_{1}, \lambda_{2}, \lambda_{3}$ the composition $L \Lambda$ is anti-symmetric.

In particular if all $\lambda$ 's are different from zero then by necessity $L \mathfrak{g} \subset \mathfrak{g}$ and $[\mathfrak{h}, \mathfrak{h}]=\mathfrak{g}$. Hence the Lie algebra $\mathfrak{h}$ must be unimodular.

By the proof of Proposition 4.1 the vector field $E=b$ satisfies (W2) if and only if $L$ is anti-symmetric on $\mathfrak{g}$. We can see that in the case when $\mathfrak{h}$ is not unimodular, $E=b$ is never a Killing vector field.

When $\mathfrak{h}$ is unimodular we get that $E=b$ satisfies (W2) if and only if it is parallel. If we assume $L \neq 0$ then it happens only for special cases: $\lambda_{1}=\lambda_{2}=\lambda_{3}$ and $L$ is an arbitrary anti-symmetric operator on $\mathfrak{g}$, or (up to permutation of indices) $\lambda_{1}=\lambda_{2} \neq \lambda_{3}$ and $L e_{1}=a e_{2}, L e_{2}=$ $-a e_{1}, L e_{3}=0$. We can thus see that parallel vector fields are found on nonabelian groups with non-flat left invariant metrics.

All the examples of SNP left invariant vector fields described above in the 3 and 4 dimensional cases of unimodular groups are parallel. This is in agreement with our general result

Theorem 4.3. For a unimodular Lie group $G$ if a left invariant vector field $E \in \mathfrak{g}$ satisfies properties (W1) and (W2) of Proposition 3.1 then it is parallel.

It follows that on a unimodular group a left invariant field $E \neq 0$ is SNP if and only if it is parallel. We will prove this theorem in the more general setting of a homogeneous Riemannian manifold in Section 7. Before that we will introduce an interesting class of multidimensional examples. They will show in particular that the assumption of unimodularity in Theorem 4.3 is essential. There are SNP vector fields on non-unimodular groups which are not Killing.

\section{Non-Positive Weyl COnnections on EXtensions of ABELIAN GROUPS}

Let us consider an $n+1$ dimensional extension $\mathfrak{s}$ of an abelian $n$ dimensional Lie-algebra $\tilde{\mathfrak{s}}$ defined by an operator $L: \tilde{\mathfrak{s}} \rightarrow \tilde{\mathfrak{s}}$. We introduce a scalar product into $\mathfrak{s}$, and let $b \in \mathfrak{s}$ be a unit vector orthogonal to $\tilde{\mathfrak{s}}$. We put $a d_{b}(u)=[b, u]=L u, u \in \tilde{\mathfrak{s}}$, and we extend naturally the operator $L$ to the whole Lie algebra $\mathfrak{s}$. The Lie algebra $\mathfrak{s}$ is unimodular if and only if the trace of $L$ vanishes. 
We restrict our attention to the case of the symmetric operator $L$ with the basis of eigenvectors $e_{i}, i=0,1, \ldots, n$ and respective eigenvalues $\mu_{i}, i=0,1, \ldots, n$, where $e_{0}=b$ and $\mu_{0}=0$,

The formulas for the covariant derivative of left invariant fields in this case can be found in [Mi]. We can summarize them as

$$
\begin{aligned}
& \nabla_{b} b=0, \nabla_{b} u=0, \text { for } u \in \tilde{\mathfrak{s}}, \\
& \nabla_{u} b=-L u, \nabla_{u} v=\langle L u, v\rangle b, \text { for } u, v \in \tilde{\mathfrak{s}}
\end{aligned}
$$

For an orthonormal frame $(X, Y)$ in $\mathfrak{s}, X=\sum_{i=0}^{n} x_{i} e_{i}, Y=\sum_{i=0}^{n} y_{i} e_{i}$, we get the Riemannian sectional curvature $K(X, Y)$

$$
\begin{aligned}
& K(X, Y)=-x_{0}^{2}\langle L Y, L Y\rangle-y_{0}^{2}\langle L X, L X\rangle+2 x_{0} y_{0}\langle L X, L Y\rangle \\
& -\langle L X, X\rangle\langle L Y, Y\rangle+\langle L X, Y\rangle^{2}= \\
& -\sum_{i=1}^{n} \mu_{i}^{2}\left(x_{0} y_{i}-y_{0} x_{i}\right)^{2}-\sum_{j<k}^{n} \mu_{j} \mu_{k}\left(x_{j} y_{k}-y_{j} x_{k}\right)^{2} .
\end{aligned}
$$

Further for a vector field $E=\gamma \tilde{E}=\gamma \sum_{i=0}^{n} \eta_{i} e_{i}, \gamma>0,|\tilde{E}|=1$, we have the following expression for the Weylian sectional curvature $\widehat{K}(X, Y)$

$$
\begin{aligned}
& \widehat{K}(X, Y)-K(X, Y)= \\
& \gamma\left(\eta_{0}(\langle L X, X\rangle+\langle L Y, Y\rangle)-x_{0}\langle L X, \tilde{E}\rangle-y_{0}\langle L Y, \tilde{E}\rangle\right) \\
& -\gamma^{2}\left(1-\langle X, \tilde{E}\rangle^{2}-\langle Y, \tilde{E}\rangle^{2}\right)
\end{aligned}
$$

Equipped with these formulas we search for non-positive homogeneous Weyl connections on the Lie group with the Lie algebra $\mathfrak{s}$.

Let the eigenvalues of $L$ be ordered as $\mu_{1} \leq \mu_{2} \leq \cdots \leq \mu_{n}$ and let us assume that all these eigenvalues are different from zero (apart from $\left.\mu_{0}=0\right)$.

Theorem 5.1. The Weyl connection defined by $E=\alpha b$ is non-positive if and only if

(1) for $\mu_{1}<0<\mu_{n}$ :

$\alpha=\mu_{1}$ and all the negative eigenvalues are equal to $\mu_{1}$, or $\alpha=\mu_{n}$ and all the positive eigenvalues are equal to $\mu_{n}$.

(2) for $\mu_{1}>0\left(\mu_{n}<0\right)$ :

$\alpha \leq \mu_{1}\left(\alpha \geq \mu_{n}\right)$.

Proof. The sectional curvature can be viewed as a restriction of a quadratic form on the exterior square $\mathfrak{s} \wedge \mathfrak{s}$.

We will take advantage of the fact that in our special case $E=\alpha b$ the quadratic form defined by $\widehat{K}$ is "diagonal". It follows from the following 
Lemma 5.2. For two unit orthogonal vectors $X=\sum_{i=0}^{n} x_{i} e_{i}$ and $Y=$ $\sum_{i=0}^{n} y_{i} e_{i}$ we have for $k=0,1, \ldots, n$

$$
x_{k}^{2}+y_{k}^{2}=\sum_{j \neq k} m_{k j}^{2}
$$

where $m_{i j}=x_{i} y_{j}-x_{j} y_{i}$.

Proof. We will prove the formula for $k=0$. Let $\Pi$ be the orthogonal projection onto the subspace orthogonal to $e_{0}$. We compare the length of $X \wedge Y$ and $\Pi X \wedge \Pi Y$. We have $\Pi X \wedge \Pi Y=\sum_{0<i<j} m_{i j} e_{i} \wedge e_{j}$ so $|\Pi X \wedge \Pi Y|^{2}=\sum_{0<i<j} m_{i j}^{2}$. On the other hand we have for any two vectors $U, V|U \wedge V|^{2}=U^{2} V^{2}-\langle U, V\rangle^{2}$. Since the unit vectors $X, Y$ are orthogonal we have $\langle\Pi X, \Pi Y\rangle=-x_{0} y_{0}$ and it follows that

$$
\begin{aligned}
& |\Pi X \wedge \Pi Y|^{2}=|\Pi X|^{2}|\Pi Y|^{2}-\langle\Pi X, \Pi Y\rangle^{2} \\
& =\left(1-x_{0}^{2}\right)\left(1-y_{0}^{2}\right)-x_{0}^{2} y_{0}^{2}=1-x_{0}^{2}-y_{0}^{2}
\end{aligned}
$$

Since $1=|X \wedge Y|^{2}=\sum_{0<i<j} m_{i j}^{2}+\sum_{s} m_{0 s}^{2}$ we obtain the desired formula.

Using this lemma we will express $\widehat{K}(X, Y)$ as a sum of squares of $m_{i j}, i, j=0,1, \ldots, n$. The coefficient with $m_{0 k}^{2}, k>0$, is equal to $\mu_{k}\left(\alpha-\mu_{k}\right)$. The coefficient with $m_{i j}, 0<i<j$, is equal to $-(\alpha-$ $\left.\mu_{i}\right)\left(\alpha-\mu_{j}\right)$. The non-positivity of the Weyl connection is equivalent to the non-positivity of all these coefficients. We obtain readily the desired conclusions.

Theorem 5.3. If the Weyl connection defined by a left-invariant vector field $E=\sum_{i=0}^{n} \alpha_{i} e_{i}, \in \mathfrak{s}$ is non-positive then $\alpha_{0} \notin\left(\mu_{1}, \mu_{n}\right)$.

If further $\mu_{n}>0\left(\mu_{1}<0\right)$ and $\alpha_{0} \geq \mu_{n}\left(\alpha_{0} \leq \mu_{1}\right)$ then all of the positive (negative) eigenvalues of $L$ are equal to $\mu_{n}\left(\mu_{1}\right)$, and $E=\mu_{n} e_{0}$ $\left(E=\mu_{1} e_{0}\right)$.

Proof. The proof consists of four steps. We put $E=\gamma \tilde{E}=\gamma \sum_{i=0}^{n} \eta_{i} e_{i}, \gamma>$ $0,|\tilde{E}|=1$, so that $\alpha_{0}=\gamma \eta_{0}$.

Step 1. $\alpha_{0} \notin\left(\mu_{1}, \mu_{n}\right)$

To prove it we will be choosing an orthonormal frame $(X, Y)$ in $\tilde{\mathfrak{s}}$ such that $E-\alpha_{0} e_{0} \in \tilde{\mathfrak{s}}$ belongs to the plane spanned by $(X, Y)$ and $\langle L X, Y\rangle=0$. The Weylian sectional curvature in the direction of this plane is by (9) and (10) equal to

$$
\begin{aligned}
& \widehat{K}(X, Y)=-\left(\gamma \eta_{0}\right)^{2}+\gamma \eta_{0}(\langle L X, X\rangle+\langle L Y, Y\rangle)+\langle L X, X\rangle\langle L Y, Y\rangle \\
& =-\left(\gamma \eta_{0}-\langle L X, X\rangle\right)\left(\gamma \eta_{0}-\langle L Y, Y\rangle\right) .
\end{aligned}
$$

We conclude that if the Weyl connection is non-positive then $\gamma \eta_{0} \leq$ $\langle L X, X\rangle$ or $\gamma \eta_{0} \geq\langle L Y, Y\rangle$. In other words $\gamma \eta_{0}$ does not belong to the open interval $(\langle L X, X\rangle,\langle L Y, Y\rangle)$. 
Assuming $\sum_{j=2}^{n} \eta_{j} e_{j} \neq 0$, we choose $X=e_{1}$ and $Y=\sigma_{1} \sum_{j=2}^{n} \eta_{j} e_{j}$, where $\sigma_{1}$ is the normalizing factor $\sigma_{1}^{-2}=1-\eta_{0}^{2}-\eta_{1}^{2}$.

We get that $\gamma_{0} \eta_{0}$ does not belong to the interval $\left(\mu_{1}, b\right)$ where $b$ is a weighted average of the eigenvalues $\mu_{2}, \ldots, \mu_{n}$. More precisely $b=\sigma_{1}^{2}\left(w+\eta_{n}^{2} \mu_{n}\right)$ where $w=\sum_{i=2}^{n-1} \eta_{i}^{2} \mu_{i}$.

Similarly, if $\sum_{j=1}^{n-1} \eta_{j} e_{j} \neq 0$, we choose the orthonormal frame $(X, Y)$, where $X=e_{n}$ and $Y=\sigma_{n} \sum_{j=1}^{n-1} \eta_{j} e_{j}, \sigma_{n}^{-2}=1-\eta_{0}^{2}-\eta_{n}^{2}$. We arrive at the conclusion that $\gamma \eta_{0}$ does not belong to the open interval $\left(a, \mu_{n}\right)$ where $a=\sigma_{n}^{2}\left(\eta_{1}^{2} \mu_{1}+w\right)$. It can be checked that $a<b$. It follows that $\gamma \eta_{0}$ does not belong to the open interval $\left(\mu_{1}, \mu_{n}\right)$.

It remains to analyze the remaining cases of $\sum_{j=2}^{n} \eta_{j} e_{j}=0$, and/or $\sum_{j=1}^{n-1} \eta_{j} e_{j}=0$. In both cases we choose $X=e_{1}, Y=e_{n}$ to arrive at the same conclusion.

Step 2. If $\mu_{1}<0\left(\mu_{n}>0\right)$ and $\alpha_{0} \leq \mu_{1}\left(\alpha_{0} \geq \mu_{n}\right)$ then $\alpha_{0}=\mu_{1}$ $\left(\alpha_{0}=\mu_{n}\right)$, and if $\mu_{k}>\mu_{1}\left(\mu_{k}<\mu_{n}\right)$ then $\alpha_{k}=0$.

Let us assume without loss of generality that $\alpha_{0}=\gamma \eta_{0} \leq \mu_{1}<$ 0 . We choose an orthonormal frame $(X, Y)$ where $X=e_{1}, Y=$ $\sigma\left(\eta_{0} e_{0}+\sum_{i=2}^{n} \eta_{i} e_{i}\right)$ and $\sigma$ is the normalizing factor $\sigma^{-2}=1-\eta_{1}^{2}$. We have that $E$ belongs to the plane spanned by $X$ and $Y$. The Weylian curvature in the direction of this plane is equal by (9) and (10) to

$$
\begin{aligned}
& \widehat{K}(X, Y)=-\sigma^{2} \eta_{0}^{2} \mu_{1}^{2}-\sigma^{2} \mu_{1} \sum_{i=2}^{n} \mu_{i} \eta_{i}^{2}+\gamma \eta_{0} \mu_{1}= \\
& -\mu_{1}^{2}+\sigma^{2} \sum_{i=2}^{n}\left(\mu_{1}^{2}-\mu_{1} \mu_{i}\right) \eta_{i}^{2}+\gamma \eta_{0} \mu_{1} .
\end{aligned}
$$

The non-positivity of the curvature implies that

$$
\mu_{1}^{2} \leq \gamma \eta_{0} \mu_{1} \leq \mu_{1}^{2}-\sigma^{2} \sum_{i=2}^{n}\left(\mu_{1}^{2}-\mu_{1} \mu_{i}\right) \eta_{i}^{2} \leq \mu_{1}^{2} .
$$

It follows that $\sum_{i=2}^{n}\left(\mu_{1}^{2}-\mu_{1} \mu_{i}\right) \eta_{i}^{2}=0$ and $\gamma \eta_{0}=\mu_{1}$. We have thus achieved Step 2.

Step 3. If $\alpha_{0}=\mu_{1}<0$ then $E=\mu_{1} e_{0}$.

We have by step 2 that $E=\gamma \sum_{i=0}^{k} \eta_{i} e_{i}$ and $\mu_{1}=\mu_{2}=\cdots=\mu_{k}<0$. We choose an orthonormal frame $(X, Y)$ where $X=x_{0} e_{0}+x_{1} e_{1}$ with arbitrary coefficients $x_{0}, x_{1}, x_{0}^{2}+x_{1}^{2}=1$, and $Y=\sigma \sum_{j=2}^{k} \eta_{j} e_{j}$, where $\sigma^{-2}=1-\eta_{0}^{2}-\eta_{1}^{2}$. If $E=\alpha_{0} e_{0}+\alpha_{1} e_{1}$ then we take $Y$ to be an arbitrary unit vector orthogonal to all the vectors $X$. The Weylian sectional curvature $\widehat{K}(X, Y)$ can be represented as a quadratic form in the variables $\left(x_{0}, x_{1}\right), \widehat{K}(X, Y)=A x_{0}^{2}+B x_{0} x_{1}+C x_{1}^{2}$.

By direct calculation we get $C=0$ and $B=\mu_{1} \alpha_{1}$. It follows that $\alpha_{1}=0$. Since the status of all the components $\alpha_{1}, \alpha_{2}, \ldots, \alpha_{k}$ is the same under present assumptions, we conclude that all of them must be zero. 
Step 4. If $\mu_{1}<0$ and $E=\mu_{1} e_{0}$ then all the negative eigenvalues of $L$ are equal to $\mu_{1}$.

For $\mu_{1}<\mu_{i}<0$ we choose the orthonormal frame $X=e_{0}$ and $Y=e_{i}$. We get that $\widehat{K}(X, Y)=-\mu_{i}^{2}+\mu_{1} \mu_{i}>0$. This contradiction ends the proof of Step 4, and the proof of the Theorem.

The last theorem gives us immediately the following

Corollary 5.4. If $L$ has both positive and negative eigenvalues and the Weyl connection defined by $E$ is non-positive then $E=\mu_{n} e_{0}$ and all the positive eigenvalues are equal to $\mu_{n}$, or $E=\mu_{1} e_{0}$ and all the negative eigenvalues are equal to $\mu_{1}$.

The last Corollary covers the case of a unimodular non-abelian Lie algebra $\mathfrak{s}$, since then $\sum_{i=1}^{n} \mu_{i}=0$.

Let us assume that $e^{L}$ is an automorphism of a discrete subgroup $\Gamma_{0}<\mathbb{R}^{n}$ with $n$ generators. The group $\Gamma>\Gamma_{0}$ generated by $\Gamma_{0}$ and $e^{L}$ is a discrete subgroup of the simply connected Lie group $S$ with the Lie algebra $\mathfrak{s}$. The homogeneous space $M={ }_{\Gamma} \S$ is then compact and we get a family of examples of compact manifolds with only one isolated nonpositive Weyl connection. The geodesic flows of such connections were recently investigated in [W4]. One of the conclusions is the description of the Gaussian thermostat defined on $M$ by the left invariant vector field $E=b$. As explained in the Introduction we may get different dynamics for different magnitudes of the velocity $k=v^{2}$. In the case of $E=b$ the Gaussian thermostat for $0<k<\mu_{n}^{-1}$ is asymptotic to an Anosov flow (the suspension of the hyperbolic toral automorphism $\left.e^{L}\right)$. For $k>\mu_{n}^{-1}$ an open dense subset of the phase space is foliated by invariant tori carrying quasi-periodic motions, while the Anosov subsystem on a submanifold of half the dimension of the phase space is still present. Hence the isolated non-positive Weyl connection defined by $\mu_{n} E$ corresponds to the borderline dynamics.

Finally let us analyze the case of all positive, (or all negative) eigenvalues, $0<\mu_{1} \leq \mu_{2} \leq \cdots \leq \mu_{n}$. In this case the Riemannian sectional curvatures are all negative, and hence the homogeneous Weyl connection defined by $E \in \mathfrak{s}$ is non-positive if only $\gamma=|E|$ is sufficiently small. We do not attempt to give explicit conditions for non-positivity in this case. Instead we describe all SNP vector fields $E \in \mathfrak{s}$ under the assumption that $\operatorname{div} E=0$.

For a unit vector field $E=\sum_{i=0}^{n} \eta_{i} e_{i}$ we have $\operatorname{div} E=\eta_{0} \sum_{i=0}^{n} \mu_{i}$. Hence either $\sum_{i=0}^{n} \mu_{i}=0$ and the group is unimodular, or $\sum_{i=0}^{n} \mu_{i} \neq 0$ and then div $E=0$ if and only if $E \in \widetilde{\mathfrak{s}}$ (i.e., if $E$ is orthogonal to $\left.b=e_{0}\right)$.

For a vector field $E=-e_{0}$ we get $\left\langle\nabla_{Y} E, Y\right\rangle=1$ for every unit vector $Y$ orthogonal to $E$. It follows from Proposition 3.3 that all vectors close to $-e_{0}$ are SNP. 
The divergence free vector fields $E \in \widetilde{\mathfrak{s}}$ which are SNP are described in the following

Proposition 5.5. If a unit divergence free vector field $E \in \widetilde{\mathfrak{s}}$ is $S N P$ then $E$ is an eigenvector of $L$ with the eigenvalue $\mu_{k}$ such that $\mu_{k} \leq 4 \mu_{1}$.

If $E$ is an eigenvector of $L$ with the eigenvalue $\mu_{k}$ such that $0<\mu_{k}<$ $4 \mu_{1}$ then it is $S N P$.

Proof. We apply directly Proposition 3.2. For a vector $Y=\sum_{i=0}^{n} y_{i} e_{i}$ orthogonal to $E$ we must have $\left\langle\nabla_{Y} E, Y\right\rangle=0$. By (8) we get $\nabla_{Y} E=$ $\langle L Y, E\rangle b$, and finally if $y_{0} \neq 0$ we conclude that $\langle L Y, E\rangle=\langle Y, L E\rangle=$ 0 for any vector $Y$ orthogonal to $E$. It implies that $E$ must be an eigenvector of $L$.

If $E=e_{k}$ is an eigenvector of $L$ with the eigenvalue $\mu_{k}$ then for $Y_{1}=b$ the condition (W3) says that $\mu_{k}^{2} \leq-4 K\left(E, Y_{2}\right)$ for any vector $Y_{2} \in \tilde{\mathfrak{s}}$ orthogonal to $E$. The Riemannian curvature in the plane spanned by $E$ and $Y_{2}=\sum_{i \neq k}^{n} y_{i} e_{i}$ is by (9) equal to

$$
-K\left(E, Y_{2}\right)=y_{0}^{2} \mu_{k}^{2}+\sum_{i \neq 0, k} y_{i}^{2} \mu_{i} \mu_{k}
$$

It is the smallest in absolute value for $Y_{2}=e_{1}$ and $-K\left(e_{k}, e_{1}\right)=\mu_{1} \mu_{k}$. This gives us the condition $\mu_{k} \leq 4 \mu_{1}$.

Conversely, if $\mu_{k}<4 \mu_{1}$ then the condition (W5) is satisfied and the proof is complete.

The above examples show that a unit divergence free vector field $E$ can have the SNP property without being a Killing field.

In particular if $L$ is equal to identity then we get the $(n+1)$ dimensional hyperbolic space $\mathcal{H}^{n+1}$. If we model it by the upper half space then the Lie group of isometries consists of translations and dilations, and it can be identified with $\mathcal{H}^{n+1}$.

The unit divergence free left invariant vector fields are horizontal fields which have constant direction in the Euclidean sense, e.g. it has the direction of the first axis. Clearly they do not have constant length in the Euclidean sense and they are not Killing fields. Any such vector field defines a homogeneous Weyl connection which is SNP. Let us note that this example cannot be factored onto a compact manifold. We do not know an example of a divergence free vector field on a compact manifold with the SNP property which is not parallel.

Additionally in the hyperbolic space we have that for a vector field $E=\sum_{i=0}^{n} \alpha_{i} e_{i}$ we have $\left\langle\nabla_{Y} E, Y\right\rangle=-\alpha_{0}$ for every unit vector $Y$ orthogonal to $E$.

Hence by Proposition 3.3 we have in the hyperbolic space that all vector fields $E$ with $\langle E, b\rangle<0$ are SNP. These fields are clearly not Killing vector fields. 


\section{Homogeneous Riemannian spaces}

Let $\pi: G \rightarrow G / H=M$ be a Riemannian homogeneous space. We assume that the Lie algebra $\mathfrak{g}=\mathfrak{p} \oplus \mathfrak{h}$, where $\mathfrak{h}$ is the Lie algebra of the compact subgroup $H<G$, and the splitting is $A d(H)$ invariant.

Let $e$ denote the unit element in $G$ and let $\pi(e)=o$. We can identify the tangent space $T_{o} M$ with $\mathfrak{p}$. However let us note that at any other point in $M$ we do not have in general a canonical identification of the tangent space with $\mathfrak{p}$. We choose a scalar product in $\mathfrak{p}$ which is $A d(H)$ invariant. We spread the scalar product to other tangent spaces of $M$ by the action of the Lie group $G$ on the manifold $M=G / H$. This gives us a homogeneous Riemannian metric on $M$.

The left-invariant vector fields on $G$ do not project onto $M$. However the right-invariant vector fields do. Indeed, the action by left translations of a one parameter subgroup $g^{u}, u \in \mathbb{R}$, projects onto $M$ as a group of isometries. Its velocity field is hence a Killing vector field on $M$.

These Killing fields are projections under $\pi: G \rightarrow M=G / H$ of the right-invariant vector fields on the group $G$. Choosing an element from the Lie algebra $\mathfrak{g}$ determines uniquely the whole Killing field on $M$. The Killing field vanishes usually at some points away from the point $o$.

For Killing vector fields $X, Y, Z$ on $M$ we have the following

$$
\left\langle\nabla_{X} Y, Z\right\rangle=\frac{1}{2}(\langle[X, Y], Z\rangle-\langle[Z, X], Y\rangle-\langle[Z, Y], X\rangle)
$$

It is a completely general formula of Riemannian geometry. To apply this formula in our special setting we need to exercise some caution since the covariant derivative of a Killing vector field is not necessarily Killing. We will consider the last formula where $X, Y$ and $Z$ are projections on $M$ of right-invariant vector fields on $G$. Such vector fields project as Killing vector fields on $M$. The commutator of rightinvariant vector fields projects to $M$ as the commutator of respective projections. It allows us to obtain an explicit formula for the covariant derivative, at least at the point $o$.

We assume $Z \in \mathfrak{p}$, however we allow arbitrary right-invariant vector fields $X$ and $Y$.

We introduce the tensor $U(X, Y)$ in $\mathfrak{g}$ with values in $\mathfrak{p}$ by

$$
\langle U(X, Y), Z\rangle=\frac{1}{2}\left(\left\langle[Z, X]_{\mathfrak{p}}, Y\right\rangle+\left\langle[Z, Y]_{\mathfrak{p}}, X\right\rangle\right),
$$

where the subscript $\mathfrak{p}$ indicates the projection to $\mathfrak{p}$. At the point $O$ we get for two Killing vector fields on $M$, obtained as projections of right-invariant vector fields $X, Y$ on $G$

$$
\left(\nabla_{X} Y\right)(o)=-\frac{1}{2}[X, Y]_{\mathfrak{p}}+U(X, Y)
$$


We will need a formula for the sectional curvature on $M$. It can be written in terms of a tensor on $\mathfrak{p}$. It can be found in $\mathrm{Be}$, with a complete derivation. However we will need it in a somewhat different form, and we give an independent proof. Note the apparent asymmetry of the following formula.

Theorem 6.1. The sectional curvature $K=K(v, J)$ of $M$ in the direction of the plane spanned by an orthonormal frame $v, J \in \mathfrak{p}$ is equal to

$$
\begin{aligned}
K= & \left(\frac{1}{2}[J, v]_{\mathfrak{p}}+U(v, J)\right)^{2} \\
& -\left\langle[[J, v], v]_{\mathfrak{p}}, J\right\rangle+\left\langle[J, U(v, v)]_{\mathfrak{p}}, J\right\rangle-\left\langle[J, v]_{\mathfrak{p}},[J, v]_{\mathfrak{p}}\right\rangle
\end{aligned}
$$

Proof. We will use the Jacobi fields of the Riemannian geometry.

Let $\gamma_{t} \in G, t \in(-\epsilon, \epsilon)$, be a lift of a geodesic from $M$ to $G$, where $\gamma_{0}$ is the unit element in $G$, and $v(t)=\frac{d}{d t} \gamma_{t}$ is the velocity field along $\gamma_{t}$.

The basic observation is that the restriction of any Killing vector field to the geodesic $\gamma_{t}$ is a Jacobi field.

Let $J \in \mathfrak{p}$, be a right-invariant vector field in $G$. Its projection to $M$ is a Killing field, and its restriction to the geodesic is a Jacobi field. We will denote the resulting Jacobi field also as $J$. If $J \in \mathfrak{p}$ is a unit vector field orthogonal to $v=v(0) \in \mathfrak{p}$ then we get from the Jacobi equations the following general formula for the sectional curvature $K=K(v, J)$

$$
K=\left(\nabla_{v} J\right)^{2}-\frac{1}{2} \frac{d^{2}}{d t^{2}} J^{2} .
$$

To get the first term we can use the formula (12)

$$
\nabla_{v} J=-\frac{1}{2}[v, J]_{\mathfrak{p}}+U(v, J) .
$$

However since it only holds at the point $o$ we cannot use it directly to calculate $\frac{d^{2}}{d t^{2}} J^{2}$. This is the main stumbling block in our derivation of the formula.

We move the Jacobi vector field $J(t)=J\left(\gamma_{t}\right)$ to $\mathfrak{g}$ by left translations and denote the resulting function by $\widetilde{J}:(-\epsilon, \epsilon) \rightarrow \mathfrak{g}$. We do not claim that $\widetilde{J}$ depends only on the geodesic in $M$, it actually depends on the particular lift $\gamma_{t}$ of the geodesic. However we can safely claim that $(J(t))^{2}=(\widetilde{J}(t))^{2}$ because the left translations on $G$ project as isometries of $M$. Our task now is to differentiate the function $(\widetilde{J})^{2}$.

We will thus differentiate $\widetilde{J}(t)$ twice with respect to the real parameter $t$. In the calculation of $(\widetilde{J}(t))^{2}$ only the projection onto $\mathfrak{p}$ matters, but we can differentiate first and project later, since the differentiation is done in the linear space $\mathfrak{g}$. 
It is well known how to take the first derivative

$$
\frac{d}{d t} \widetilde{J}=[\widetilde{J}, \widetilde{v}],
$$

where $\widetilde{v}(t) \in \mathfrak{g}$ is the left translation of the velocity vector field $v(t)$ along the geodesic $\gamma_{t}$ to the Lie algebra $\mathfrak{g}$ of $G$. It is a general formula valid in a Lie group, and it holds in the whole interval $(-\epsilon, \epsilon)$. Now we differentiate the first derivative only at $t=0$. To achieve that we need yet to find $\frac{d}{d t} \widetilde{v}$ at $t=0$.

Let us consider the velocity vector field $v(t)$ as the restriction to $\gamma_{t}$ of a time dependent right-invariant vector field $\widehat{v}(t) \in \mathfrak{g}$. Namely we put $\widehat{v}=A d_{\gamma_{t}} \widetilde{v}$. We have clearly

$$
\left.\frac{d}{d t}\right|_{t=0} \widehat{v}=\left.\frac{d}{d t}\right|_{t=0} \widetilde{v}
$$

At the same time using the equations for geodesics and the formula (12) we obtain at the point $o$

$$
0=\nabla_{v} v=\frac{d}{d t} \widehat{v}_{t=0}+U(v, v)
$$

Note that we do not claim here that the right hand side $\left(\left.\frac{d}{d t}\right|_{t=0} \widehat{v}\right)$ belongs to $\mathfrak{p}$. The equality is to be understood as the equality of projections to the subspace $\mathfrak{p}$.

We obtain thus

$$
\frac{d}{d t}{ }_{\mid t=0} \widetilde{v}=-U(v, v)+r
$$

for some $r \in \mathfrak{h}$ Since the adjoint action of $H$ on $\mathfrak{p}$ is by isometries, we have that for any $r \in \mathfrak{h}$ the operator $a d_{r}: \mathfrak{p} \rightarrow \mathfrak{p}$ is skew-symmetric. Since at $t=0$ we have $\widetilde{J}=J \in \mathfrak{p}$ then it follows that

$$
\langle[\widetilde{J}, r], \widetilde{J}\rangle=\langle[J, r], J\rangle=0 .
$$

Finally we have at $t=0$

$$
\begin{aligned}
& K(v, J)-\left(\frac{1}{2}[J, v]_{\mathfrak{p}}+U(v, J)\right)^{2}=-\frac{d}{d t}\left\langle[\widetilde{J}, \widetilde{v}]_{\mathfrak{p}}, \widetilde{J}\right\rangle= \\
& -\left\langle[[J, v], v]_{\mathfrak{p}}, J\right\rangle+\left\langle[J, U(v, v)]_{\mathfrak{p}}, J\right\rangle-\left\langle[J, v]_{\mathfrak{p}},[J, v]_{\mathfrak{p}}\right\rangle,
\end{aligned}
$$

and the desired formula follows.

\section{Homogeneous Weyl CONNECTIONS ON HOMOGENEOUS SPACES}

To introduce homogeneous Weyl connections on the Riemannian homogeneous space $G / H$ of Section 6 we consider the subspace

$$
\mathfrak{p}_{0}=\{X \in \mathfrak{p} \mid[Y, X]=0 \quad \forall Y \in \mathfrak{h}\},
$$

on which the action of $\operatorname{Ad}(H)$ is trivial.

We choose a left invariant vector field $E$ defined by an element from $\mathfrak{p}_{0}$. It projects onto $M=G / H$ as a $G$-invariant vector field. The 
subspace $\mathfrak{p}_{0}$ may be equal to zero, however for $\mathfrak{h}=0$ all of $\mathfrak{g}$ is in $\mathfrak{p}_{0}$. The last case is that of a left invariant metric on $G$, with a left invariant vector field $E$.

The Weyl connection defined on $M=G / H$ by the projection of a left-invariant vector field $E \in \mathfrak{p}_{0}$ is clearly preserved by the isometric action of the group $G$ on $M$.

Our main result says

Theorem 7.1. If the Lie group $G$ is unimodular and a $G$-invariant vector field on $M=G / H$, defined by $E \in \mathfrak{p}_{0}$, is SNP then the vector field is parallel.

We will prove a slightly more general fact.

Theorem 7.2. If the Lie group $G$ is unimodular and a G-invariant vector field on $M=G / H$, defined by $E \in \mathfrak{p}_{0}$, satisfies properties (W1) and (W2) then the vector field is parallel.

Proof. Without loss of generality we can assume $E^{2}=1$

We need a formula for $\nabla_{Y} E$, where $Y \in \mathfrak{p}$ is orthogonal to $E$. The field $E$ can be naturally considered as a left-invariant vector field in $G$ which projects onto $M=G / H$. We can extend $Y$ to a right-invariant vector field in $G$ which also projects onto $M$ (as a Killing vector field). Since any left-invariant vector field commutes with any right-invariant vector field the projections of $E$ and $Y$ on $M$ also commute. We conclude that $\nabla_{Y} E=\nabla_{E} Y$ and $Y$ is a Killing vector field. At the point $o$ we can use the formula (12) to get

$$
\nabla_{E} Y=\frac{1}{2}[Y, E]_{\mathfrak{p}}+U(E, Y) .
$$

Moreover by the definition of $U$ we get

$$
\langle U(E, Y), Y\rangle=\frac{1}{2}\left\langle[Y, E]_{\mathfrak{p}}, Y\right\rangle .
$$

We conclude that

$$
\left\langle\nabla_{Y} E, Y\right\rangle=\left\langle[Y, E]_{\mathfrak{p}}, Y\right\rangle .
$$

By the last formula the condition (W2) translates into

$$
\left\langle[E, Y]_{\mathfrak{p}}, Y\right\rangle=0
$$

for any field $Y \in \mathfrak{p}$ orthogonal to $E$.

Hence the condition (W2) can be rephrased in the following way. Let $A: \mathfrak{p} \rightarrow \mathfrak{p}$ be the operator defined as the composition of the operator $a d_{E}=[E, \cdot]$ and the orthogonal projection onto the orthogonal complement of $E$. The property (W2) is now equivalent to $A$ being skew-symmetric. We have

$$
[E, Y]_{\mathfrak{p}}=A(Y)+\sigma(Y) E
$$

for some linear functional $\sigma: \mathfrak{p} \rightarrow \mathbb{R}$. 
We invoke now the formula for the sectional curvature of $M$ at the point $o$ in the plane spanned by the unit $G$-invariant vector field $v=E$ and the Killing vector field $J=Y \in \mathfrak{p}, Y$ orthogonal to $E$.

$K(E, Y)=\left(\nabla_{Y} E\right)^{2}-\left\langle[E,[E, Y]]_{\mathfrak{p}}, Y\right\rangle-\left\langle[U(E, E), Y]_{\mathfrak{p}}, Y\right\rangle-[E, Y]_{\mathfrak{p}}^{2}$

Under the condition (W2) we have further, using the fact that $a d_{\mathfrak{h}}$ vanishes on $E$,

$$
\begin{aligned}
& \left\langle[E,[E, Y]]_{\mathfrak{p}}, Y\right\rangle=\left\langle\left[E,[E, Y]_{\mathfrak{p}}\right]_{\mathfrak{p}}, Y\right\rangle=\left\langle[E, A(Y)]_{\mathfrak{p}}, Y\right\rangle= \\
& =-\left\langle[E, Y]_{\mathfrak{p}}, A(Y)\right\rangle=-\left\langle[E, Y]_{\mathfrak{p}},[E, Y]_{\mathfrak{p}}\right\rangle+\sigma(Y)^{2} .
\end{aligned}
$$

Now the formula for the curvature reads

$$
K(E, Y)=\left(\nabla_{Y} E\right)^{2}-\sigma(Y)^{2}-\left\langle[U(E, E), Y]_{\mathfrak{p}}, Y\right\rangle .
$$

Let us choose in $\mathfrak{p}$ an orthonormal basis $E, Y_{1}, Y_{2}, \ldots Y_{n-1}$.

We calculate the Ricci curvature in the direction of $E$ by summing the sectional curvatures over the chosen orthogonal basis. It follows from the condition (W1) that $\operatorname{Ricc}(E) \leq 0$.

$$
\begin{aligned}
0 & \geq \operatorname{Ricc}(E)=\sum_{i=1}^{n-1} K\left(E, Y_{i}\right)= \\
& =\sum_{i=1}^{n-1}\left(\nabla_{Y_{i}} E\right)^{2}-\sum_{i=1}^{n-1} \sigma\left(Y_{i}\right)^{2}-\sum_{i=1}^{n-1}\left\langle\left[U(E, E), Y_{i}\right]_{\mathfrak{p}}, Y_{i}\right\rangle .
\end{aligned}
$$

Invoking the unimodularity of the Lie group we have that $a d_{U(E, E)}$ is traceless. Since $a d_{\mathfrak{h}}$ preserves $\mathfrak{p}$ there is no contribution to the trace from $\mathfrak{h}$ and we conclude that

$$
\operatorname{tr} a d_{U(E, E)}=\sum_{i=1}^{n-1}\left\langle\left[U(E, E), Y_{i}\right]_{\mathfrak{p}}, Y_{i}\right\rangle+\left\langle[U(E, E), E]_{\mathfrak{p}}, E\right\rangle=0
$$

Further we observe that in view of (111) we get

$U(E, E)=-\sum_{i=1}^{n-1} \sigma\left(Y_{i}\right) Y_{i} \quad$ and $\quad\left\langle[U(E, E), E]_{\mathfrak{p}}, E\right\rangle=\sum_{i=1}^{n-1} \sigma\left(Y_{i}\right)^{2}$

Hence using this and (15) the inequality (14) becomes

$$
0 \geq \operatorname{Ricc}(E)=\sum_{i=1}^{n-1}\left(\nabla_{Y_{i}} E\right)^{2} .
$$

We conclude that for every $Y$ orthogonal to $E$

$$
\nabla_{Y} E=0 \text { and } K(E, Y)=0 .
$$

Going back to the equation (13) we obtain for every $Y \perp E$

$$
0=K(E, Y)=-\sigma(Y)^{2}-\left\langle[U(E, E), Y]_{\mathfrak{p}}, Y\right\rangle .
$$


Substituting $Y=U(E, E)$ we obtain

$$
0=\sigma(U(E, E))=-\sum_{i=1}^{n-1} \sigma\left(Y_{i}\right)^{2}
$$

and hence the functional $\sigma$ vanishes. We conclude that also $\nabla_{E} E=$ $U(E, E)=0$. We can see that $\nabla_{Y} E=0$ for every vector $Y \in \mathfrak{p}$, i.e., the vector field $E$ is parallel on $M$.

\section{REFERENCES}

[Al] Alekseevski, D. V.: Homogeneous Riemannian spaces of negative curvature. (Russian) Mat. Sb. (N.S.) 96(138) (1975), 93 - 117, 168.

[Az-W] Azencott, R., Wilson, E. N.: Homogeneous manifolds with negative curvature. I. Trans. Amer. Math. Soc. 215 (1976), 323 - 362.

[Be] Besse, A. L. Einstein manifolds. Springer-Verlag, Berlin, 2008.

[D-P] Dairbekov, N. S.; Paternain, G. P. Entropy production in thermostats. II. J. Stat. Phys. 127 (2007), $887-914$.

[F] Folland, G. B.: Weyl manifolds. J. Diff. Geom. 4, (1970), 145 - 153.

[Gau] Gauduchon, P.: La 1-forme de torsion d'une vari'et'e hermitienne compacte. Math. Ann. 267 (1984), 495 - 518.

[G-R] Gallavotti, G., Ruelle, D.: SRB states and nonequilibrium statistical mechanics close to equilibrium. Comm. Math. Phys. 190 (1997), 279-285.

[He] Heintze, E.: On homogenous manifolds of negative curvature. Math. Ann. 211 (1974), $23-34$.

[H] Hoover, W.G.: Molecular Dynamics, L.N. Phys. 258, Springer (1986).

[Ke] Kerr, M. M.: Homogeneous Einstein-Weyl structures on symmetric spaces. Ann. Global Anal. Geom. 15 (1997), $437-445$.

[K-N] Kobayashi, S.; Nomizu, K.: Foundations of differential geometry. Vol. I. (IV.5) John Wiley \& Sons, Inc., New York, 1996.

[Mi] Milnor, J.: Curvatures of left invariant metrics on Lie groups. Advances in Math. 21 (1976), 293 - 329.

[N] Narita, F.: Weyl space forms and their submanifolds. Colloq. Math. 89 (2001), $117-131$.

[P-W] Przytycki, P.; Wojtkowski, M. P.: Gaussian thermostats as geodesic flows of nonsymmetric linear connections. Comm. Math. Phys. 277 (2008), 759769 .

[T] Troyanov, M.: L'horizon de SOL. Exposition. Math. 16 (1998), $441-479$.

[W1] Wojtkowski, M.P.: W-flows on Weyl manifolds and Gaussian thermostats. J. Math. Pures Appl. 79(10) (2000), 953 - 974.

[W2] Wojtkowski, M.P.: Weyl manifolds and Gausssian thermostats. In: Proc. ICM Beijing 2002, Beijing: Higher Education Press, 2003, pp. 3511 - 3523

[W3] Wojtkowski, M.P.: Rigidity of some Weyl manifolds with nonpositive sectional curvature. Proc. Amer. Math. Soc. 133 (2005), 3395 - 3402.

[W4] Wojtkowski, M.P.: Integrability via reversibility. preprint (2015), arXiv:1502.03074.

Faculty of Mathematics and Computer Science

UNIVERSITY OF WARMIA AND MAZURY

10-710 OLSZTYN, POLAND

E-mail address: wojtkowski@matman.uwm.edu.pl 